\title{
Large-scale attribution of trend in UK flood flow data
}

\author{
T.R. Kjeldsen*, C. Svensson and J.M. Miller \\ Centre for Ecology \& Hydrology, Wallingford \\ *Email: trkj@ceh.ac.uk
}

\begin{abstract}
The objective of this study is to undertake a preliminary investigation of trend in annual maximum series of peak flood data in the United Kingdom, as a precursor to developing a more complete procedure for non-stationary flood frequency estimation. A fifth of the trends in series that are at least 20 years long are significant at the 5\% level (of in total 388 series). Most of the significant trends are positive, and are located in the north and west. The largest positive trends occur for short records in the most recent decades. Trends were also investigated for various subsets of the data, based on different catchment characteristics. There is an indication that the range of trend values observed for urban catchments is larger than the range observed for the rural subset, and that storage of water, whether in lakes and reservoirs or in permeable geology, has an ameliorating effect on trend magnitude.
\end{abstract}

\section{Introduction}

Estimates of expected extreme flood events are required for design and operation of vital infrastructure such as flood defences, bridges and culverts, and also for more general flood risk management and planning, e.g. emergency planning, flood risk mapping, and for defining flood insurance premiums. In the UK, this information is obtained primarily through the use of flood frequency estimation techniques as described in the Flood Estimation Handbook (FEH) and its subsequent developments. The methods are based on the principle of analysing series of observed events to infer a probabilistic behaviour, which is then extrapolated to provide estimates of the likely magnitude of extreme events (e.g. the magnitude of the flood expected to be exceeded on average once every 100 years). The statistical models underpinning current procedures are built on the assumption of a stationary underlying distribution of the flood series.

There is much discussion in the scientific and public domain as to the impact of environmental change on the characteristics of extreme flood events (Hirsch, 2011). Numerous studies based on downscaling of future projections from global or regional climate models have reported possible future changes of flood quantiles, resulting in a $20 \%$ sensitivity allowance for testing of flood designs against projected changes in peak flows (e.g. Wilby et al., 2008). Identification of a general upward trend in data series of flood events observed in UK catchments have so far been more elusive. Based on analysis of the 1000 annual maximum series (AMS) of peak flow used in the development of the FEH, Robson (2002) concluded that there was no evidence of trend in UK flood behaviour. From a study of trend in runoff series from 87 undisturbed benchmark catchments, Hannaford and March (2008) found evidence of upward trend in flood data in maritime-influenced, upland catchments in the north and west of the UK, but no compelling evidence for trends in flood series in lowland areas in the south and east of the country. Interestingly, their study concluded that there were fewer trends in flood magnitude, as measured by the instantaneous AMS than in more aggregated indicators such as the maximum 10- or 30-day flow. In a detailed study of flood flow and water levels in the river Thames, Marsh and Harvey (2012) found no increase in peak flow over the last decade, and even a decrease in maximum flood levels resulting from increased flood management interventions, thus highlighting the complex interactions between natural and man-made interventions in the flow regime.

The impact of land-use change on flood characteristics has been studied in a range of UK catchments. There is still an active scientific debate as to the importance of land-use effects on flood runoff. However, there is generally consensus that urban development (increasing impervious areas and improving drainage systems) can have a profound effect on the hydrological processes (e.g. Wheater and Evans, 2009). Based on analysis of AMS of peak flow from 200 catchments with significant urban land-cover from the Environment-Agency's HiFlows-UK database, Kjeldsen (2010) reported a higher flood magnitude than would have been expected if the catchments were rural; though in some cases a decrease in flood magnitude was found.

The objective of this study is to undertake a preliminary investigation of trend in a contemporary UK dataset of annual maximum series (AMS) of peak flood data, as a precursor to developing a more complete procedure for non-stationary flood frequency estimation. For the purpose of screening the national dataset, an analytically tractable framework was adopted based on a two-parameter log-normal distribution with an assumed trend in the location parameter only. This involves applying a linear regression analysis to the log-transformed AMS to estimate the trend. Although this has the disadvantage of potentially exacerbating the estimated trend (as discussed later) compared with trends in untransformed data, it has the advantage of providing a convenient framework for extrapolation (in time) of the flood frequency estimates.

\section{Methodology}

The probability model adopted in this study for exploring trend in AMS of peak flows, $Q^{t}$, is adopted from Vogel et al. (2011) and assumes that the underlying distribution of 
the AMS is a two-parameter log normal (LN2) distribution. The LN2 distribution has previously been found to provide a reasonable fit to UK flood data (Laio et al., 2009). Assuming a stationary flood series, the LN2 quantile function, $q_{r}$, is defined for a return period $\mathrm{T}$ as

$$
q_{r}=\exp \left(\mu_{x}+z_{T} \sigma_{x}\right.
$$

where $\mu_{\mathrm{x}}$ and $\sigma_{x}$ are mean and standard deviation of the log transformed annual maximum flow $x_{t}=\ln \left(Q_{t}\right)$, and $z_{T}$ is the standard normal variate with an exceedance probability defined as $p=1 / T$. A simple extension of the stationary LN2 model was proposed by Vogel et al. (2011) assuming that only the mean of the LN2 distribution will change over time, and that the standard deviation remains constant, i.e.

$$
q_{T}(t)=\exp \left(\mu_{x}(t)+z_{T} \sigma_{x}\right)
$$

Here, the mean value of the log-transformed AMS is considered to be a time dependent parameter described by a linear model as

$$
\mathrm{xt}=\ln \left(Q_{t}\right)=\alpha_{0}+\alpha_{1} t+\varepsilon_{t}
$$

where $t$ is an index of the water-year in which the annual maximum peak flow is recorded, $\alpha_{0}$ and $\alpha_{1}$ are model parameters and $\varepsilon_{t}$ is the regression model error. The model parameters can be estimated using a maximum likelihood scheme which might be necessary if a more complex frequency model other than LN2 was selected. However, as predictions made using the regression model is the conditional mean, Eq. (3) can be estimated separately and subsequently combined with Eq. (2) as $\mu_{x}(t)$. A simple index, $M$, of trend in the flood quantiles can then be developed by considering the ratio (or magnification factor) between the T-year event derived at two times separated by $\Delta t$ years apart as

$$
M=\frac{q_{T}(t+\Delta t)}{q_{T}(t)}=\exp \left(\alpha_{\mathrm{i}} \Delta t\right)
$$

where $\alpha_{1}$ is the trend of the log-linear regression model in Eq. (3) and the time period $\Delta t$ is defined in this study as 10 years, i.e. $\mathrm{M}$-values can be interpreted as a decadal magnification factor (Vogel et al., 2011).

\section{The HiFlows-UK dataset}

The flood data used in this study come from the HiFlows-UK dataset v.3.1.1 and consist of annual maximum series (AMS) of peak flow up to, and including, the water year 2009 from 943 UK catchments. Selecting only AMS classified by the gauging authorities as being both 'suitable for QMED' and 'suitable for Pooling', reduced the dataset from 943 to 590 catchments. For the subsequent trend studies, a minimum record length of 20 years was imposed, further reducing the dataset to 549 catchments. These have a mean record length of 41 years, and the longest record consists of 126 annual maxima (gauging station 39001, the Thames at Kingston). A summary of the dataset is shown in Figure 1.

For each catchment, a set of FEH catchment descriptors were extracted that are typically used in current FEH procedures for flood frequency estimation. These include catchment area (AREA) in $\mathrm{km}^{2}$; standard annual average rainfall as measured from 1961 to 1990 (SAAR) in mm; an index related to the hydrological properties of catchment soils (BFIHOST), which can take on values between zero (impermeable) and one (completely permeable); an index of flood attenuation due to reservoirs and lakes (FARL), which

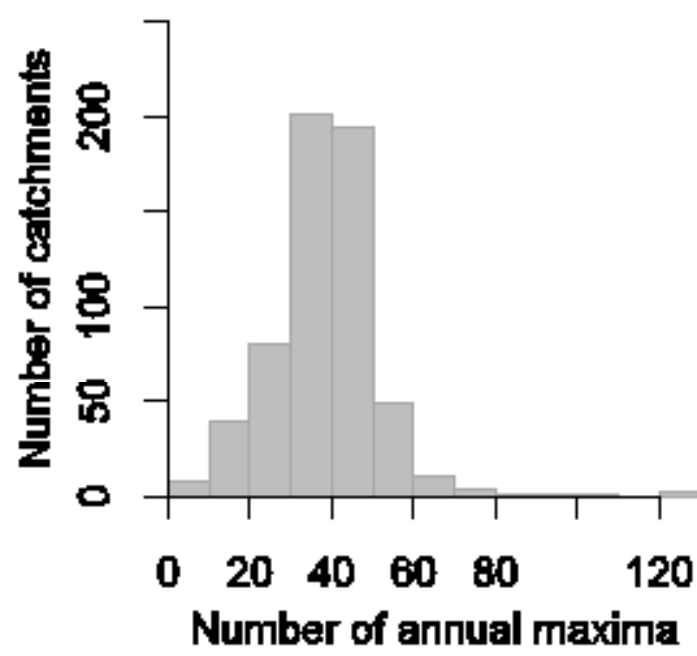

Figure 1 Histogram showing the number of annual maxima in 590 catchments in the HiFlows-UK database, which are considered suitable for both QMED estimation and inclusion in pooling groups for regional flood frequency estimation.

varies between zero and one ( 1 = no attenuation); and finally, the proportion of urban land-cover (URBEXT2000) where the subscript refers to digital land-cover maps representing the catchment state between 1998 and 2000.

A further subset of the 549 catchments was defined consisting of 69 gauging stations classified as benchmark catchments. These catchments are deemed to have a nearnatural flow regime, with little impact of human activity (Hannaford and Marsh, 2008).

\section{Results}

Adopting an LN2 distribution as default, the trend model in Eq. (3) was fitted to all the 549 AMS considered suitable for QMED and pooling and with 20 or more available annual maximum peak flow values. A Shapiro-Wilk test for normality was applied to each set of residuals to determine if the fitted trend model could be considered a reasonable model. Adopting a significance level of $5 \%$ for the normality test, a further 161 data series were rejected from the analysis on grounds of not complying satisfactorily with the normality assumption, reducing the number of catchments in the analysis to 388 . For each of the 388 data series, the decadal magnification factor, M, was estimated as shown in Eq. (4) and plotted on the map in Figure 2 at the location of the associated gauging station. The map shows that most of the fitted trend models suggest no significant (at the 5\% level) or a modest amount of trend, with a few individual catchments showing relatively large upward and, to a lesser degree, downward trends. In total 80 of the 388 catchments have significant trends.

There would not appear to be much evidence for any spatially coherent clusters of catchments with either positive or negative trends, although most of the significant trends are positive. The location of these roughly agrees with the results of Hannaford and Marsh (2008) who found positive trends in floods mainly in the northern half of the country and in the west.

It should be noted that the fitted trend models are for individual sites, and the existence of inter-site dependence in the AMS, which can influence the test statistics for significance in the trend magnitudes (Douglas and Vogel, 2000), is not considered. Consequently, the results presented in Figure 2 should not be interpreted as a formal test for the 


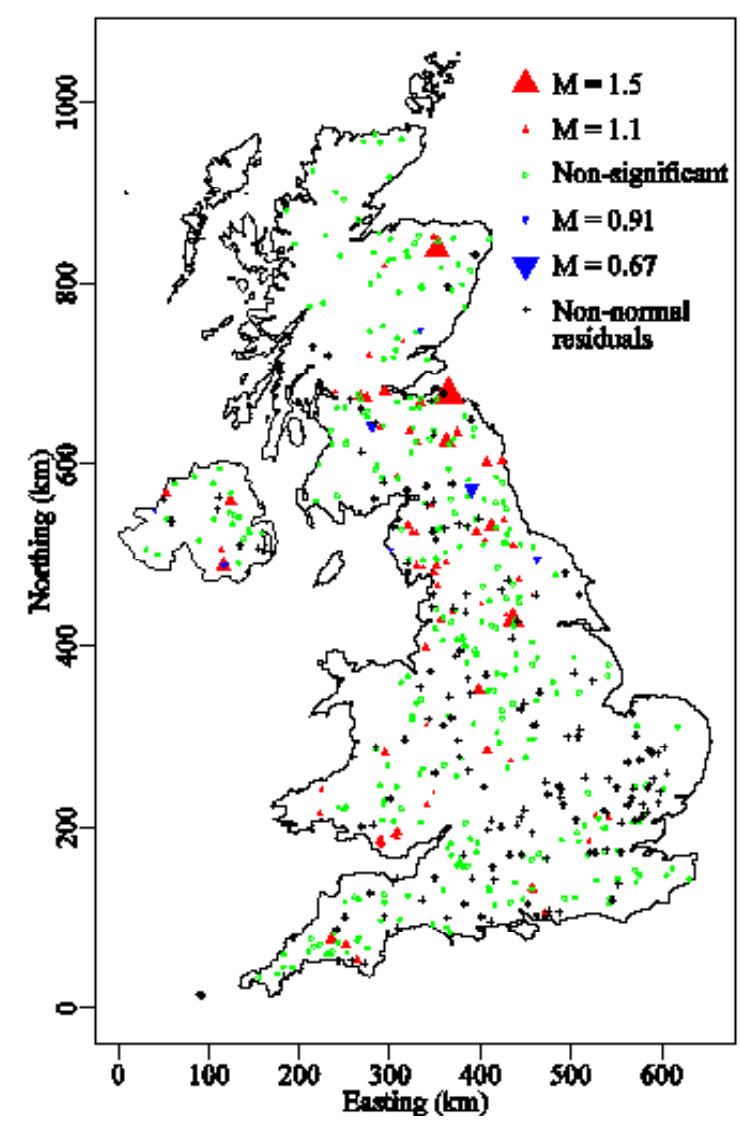

Figure 2 Estimated decadal magnification factor, M, for 388 AMS of peak flow from the HiFlows-UK database.

existence or not of significant regional patterns of trends in the flood data.

\section{Attribution of trend in AMS}

As a first attempt to attribute the presence of trends in the observed AMS to various underlying process drivers, the 388 catchments were classified based on a selection of FEH catchment descriptors which might be expected to have an influence on the catchment flood regime: URBEXT2000, FARL, BFIHOST, SAAR and AREA. For each catchment characteristic the dataset was divided into two subsets, and the distribution of M-values within and between each class is compared visually using boxplots (Figure 3 ). The two subsets for each class were defined using the threshold value shown in parenthesis in the title for each boxplot. The distribution of $M$-values from the UK benchmark catchments is also shown in each boxplot. The benchmark catchments represent the distribution of $M$-values that could be expected in catchments where little or no man-made change has occurred over the period of record. In addition to catchment descriptors, the dataset was also split according to record length to provide a better understanding of the impact of record length on the trend estimates.

The distribution of the $M$-values from all the 388 catchments ranges from 0.78 (negative trend) up to 1.61 (positive trend), with a median value of 1.039 . Hence, defining trends according to the estimate $M$ suggests a bias towards positive trends in the bulk of the AMS (however, note that most of these are not significant). The range for the benchmark catchments is nearly as wide as for the whole dataset, and the median and the range between the upper and lower quartiles of the data are also very similar. In general, where the boxplots suggest differences in the median for the subsets of the different classes (URBEXT, SAAR, etc.), these are not likely to be statistically significant and the below discussion should be taken only as a starting point for further investigations.

It can be seen from Figure 3 that the range of trend magnitudes (including direction) is wider in the Urban subset compared with the Rural within the URBEXT2000 class. A further subdivision of the Urban/Rural catchments on SAAR, into Wet and Dry catchments (Figure 4), indicates that large positive trends are mainly found in catchments that are both Urban and Dry. The Dry catchments (SAAR $<800 \mathrm{~mm}$ ) tend to be located in the lowland south and east of the country, which has a more continental climate with a higher proportion of convective rainfall than the wetter, hillier and frontal rainfall-dominated north and west. However, overall, there is an indication that trends may be lower in the Dry catchments than in the Wet (Figure 3).

The FARL subsets indicate that heavily regulated catchments have a smaller range of trend magnitudes than unregulated ones (Figure 3). Similarly, there is an indication that the water storage capacity provided in permeable catchments also lead to a slightly less wide range of trends than are found in non-permeable catchments. The attenuation of runoff that occurs in large catchments may also lead to an amelioration in the trend magnitudes, compared with those observed in Small catchments.

Catchments with long records consisting of more than 40 annual maxima tend to show smaller trends than those with Short records consisting of between 20 and 39 maxima. Some examples of individual time series will be discussed in the next section.

\section{Large positive and negative trend estimates}

Figure 5 shows the individual time series with the largest positive and negative trend magnitudes, respectively, in the dataset. It can be seen that most of the gauges with large positive trends have relatively short records covering the most recent decades, whereas the periods of record for the negative trends are more varied. It should be noted that any trend estimate based on a linear regression method is sensitive to outliers in the beginning or end of the series, compared with, for example, the non-parametric Mann-Kendal test. In addition, the log-transform will further exacerbate the effect on the trend estimate of any low observations when these occur at the beginning of the series, so that extrapolations into the future may be very different to those estimated using the slope of a linear regression line fitted to the untransformed data in natural space (Figure 6).

Further investigations are needed to establish how the period of record and the effect of regionally consistent periods of high and low floods impact the estimates of trends, especially in the case of relatively short series from now decommissioned gauging stations.

\section{Conclusions}

This study has utilized the best available gauged high-flows annual maxima for the UK in order to examine whether there is any indication of non-stationarity. From this preliminary assessment of trend a number of observations have been made:

- Considering each catchment in turn, a fifth of the trends in annual maximum series of instantaneous flow that are at least 20 years long are significant at the 5\% level. Most of the significant trends are positive, and are located in the north and west.

- There is an indication that the range of trend values observed for urban catchments is larger than the range observed for the rural subset (when significance levels of 

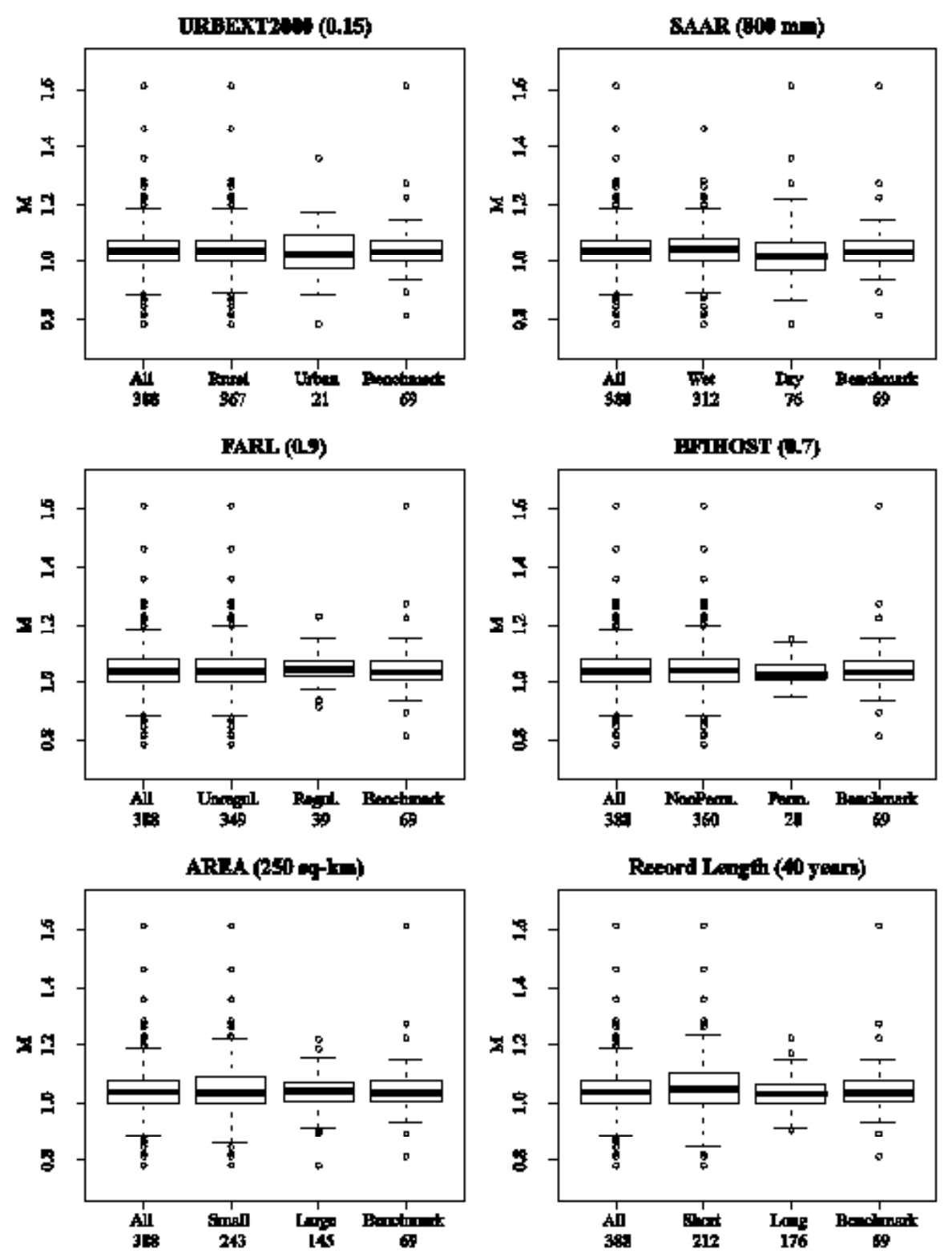

Figure 3 Boxplots comparing decadal magnification factors, $M$, between and within classes as defined by subsets of catchment descriptors. The thick line denotes the median, and the box itself outlines the upper and lower quartile. The whiskers extend to the most extreme data point which is no more than 1.5 times the length of the box away from the box, and beyond this range all individual observations are plotted.
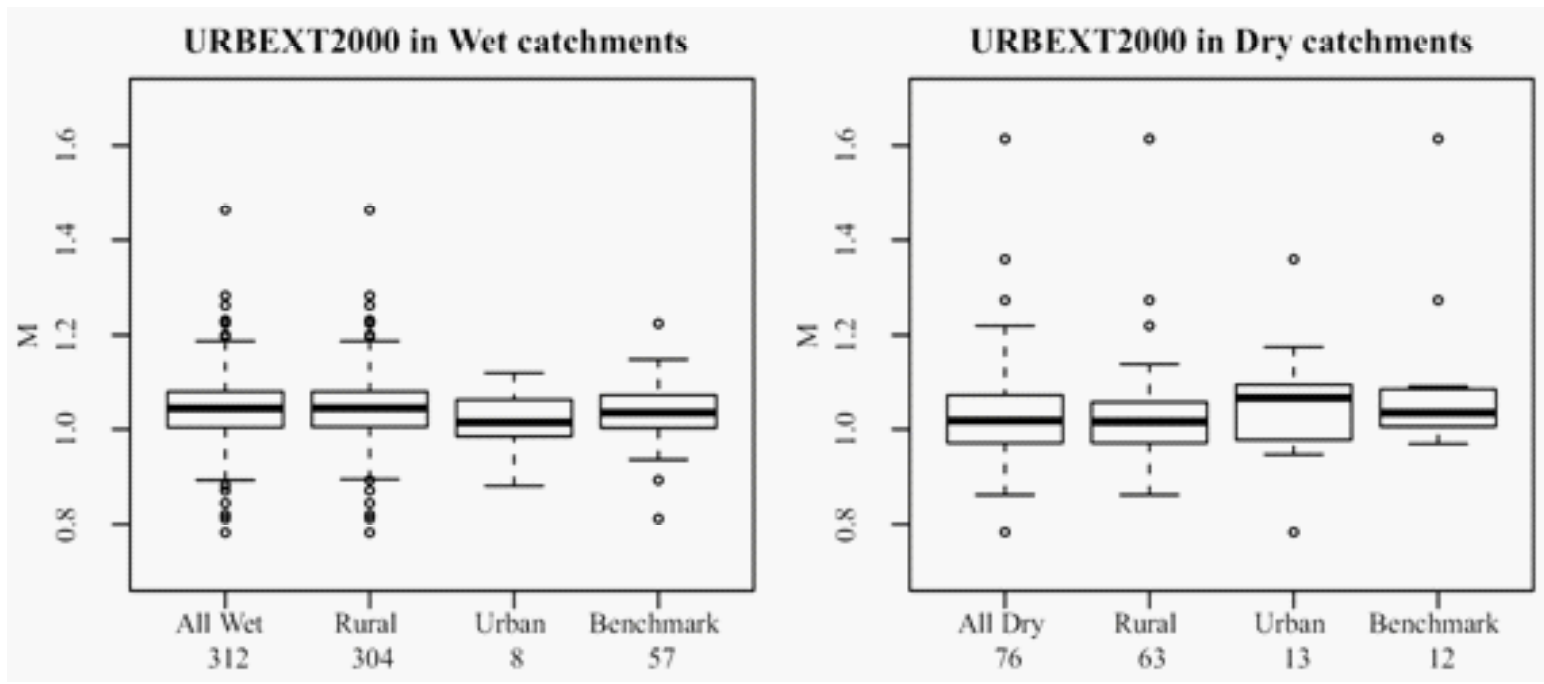

Figure 4 Boxplots comparing decadal magnification factors, M, for subsets of selected subsets shown in Figure 3. Notations as in Figure 3 with a threshold of $800 \mathrm{~mm}$ for distinguishing between Wet and Dry catchments, and of 0.15 for distinguishing between Rural and Urban catchments. 


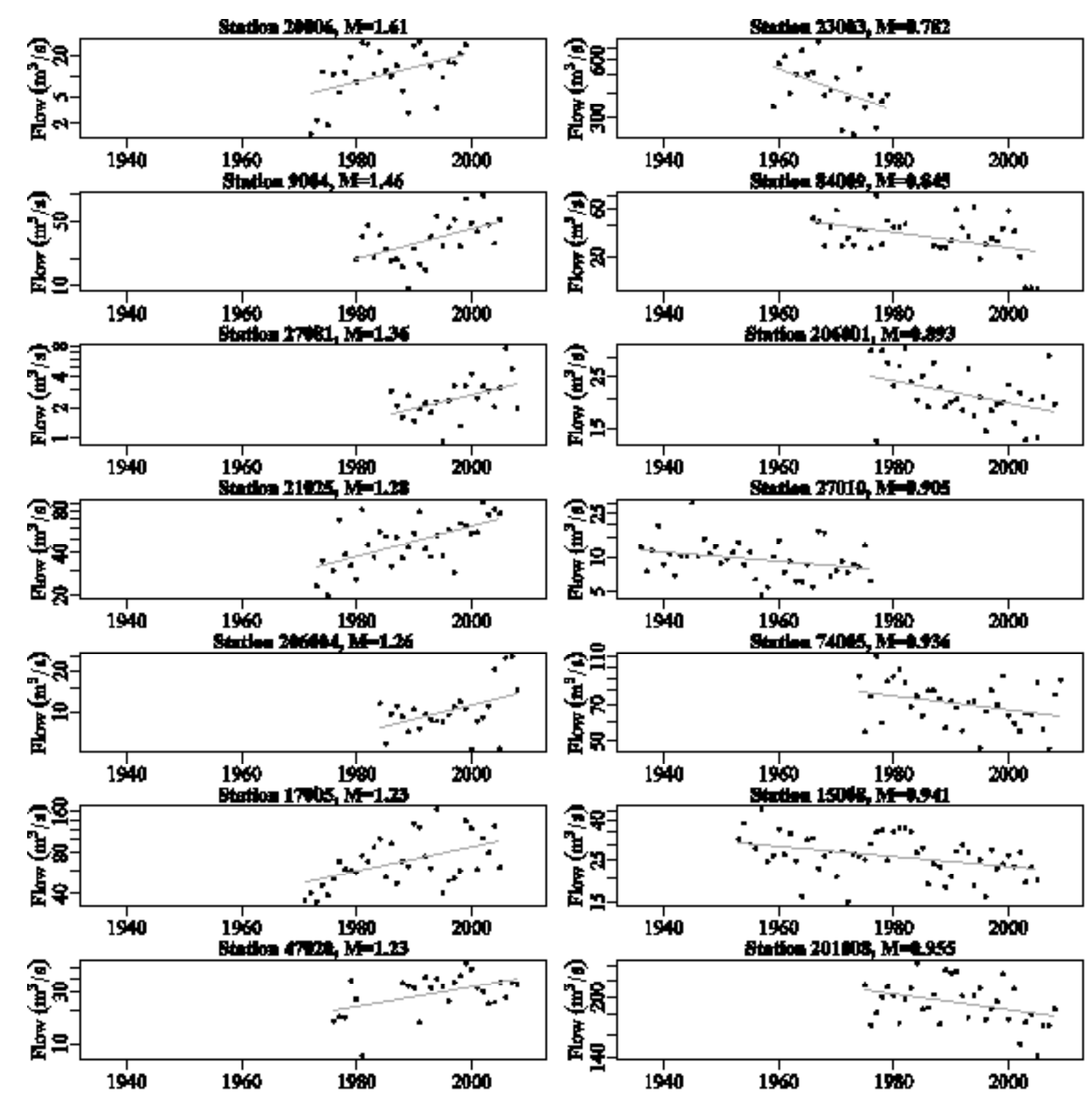

Figure 5 Time series plots of AMS for the catchments showing the largest positive (left-hand column) and negative (right-hand column) significant trends as estimated using the decadal magnification measure $M$.

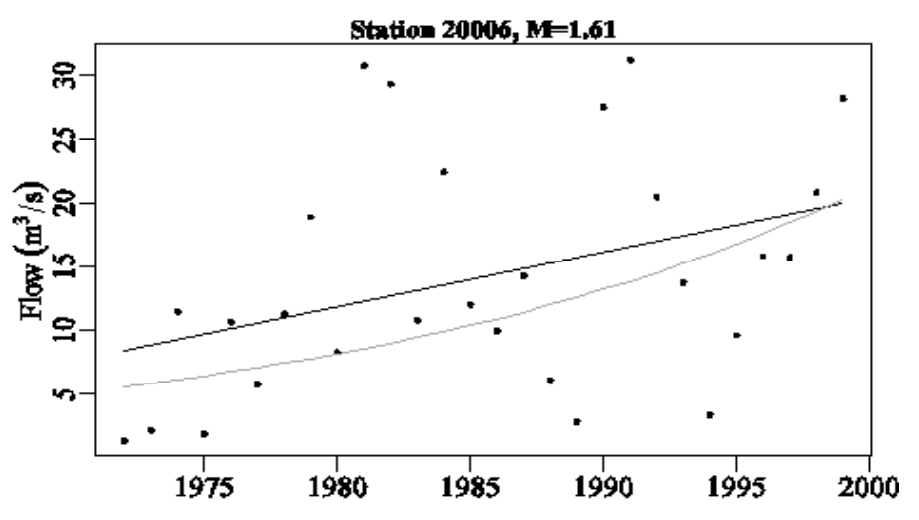

Figure 6 Time series plot of AMS for the catchment Biel Water at Belton House (gauging station 20006), and linear regression line fitted in natural space (black line) and fitted to log-transformed data (grey curved line).

the trends are not considered). This suggests that at least some changes in the flood flow regime can be attributed to urban development, and that the effects can be both a reduction and an increase in flood magnitude.
- There are indications that storage of water, whether in lakes and reservoirs or in permeable geology, has an ameliorating effect on the trend magnitude. A similar argument can be proposed for the larger catchments compared with the smaller ones, because of greater attenuation of the flow in larger catchments.

- The estimates of trend are more variable in shorter than in longer records. Most large positive trends occur for short records in the most recent decades, with a more mixed bag for the large negative trends.

- The benchmark catchments do not show a different distribution of decadal magnification values to that obtained for the dataset as a whole.

The results presented in this paper suggest that trend in AMS of peak flow can, to some degree, be linked to changing catchment conditions. Further research aimed at more detailed attribution of the changing flood characteristics is currently being undertaken by developing consistent indicators of urban change. 


\section{References}

Douglas, E.M., Vogel, RM. and Kroll, C.N. 2000. Trends in floods and low flows in the United States: impact of spatial correlation. J. Hydrol., 240, 90-105, doi:10.1016/S00221694(00)00336-X

Hannaford, J. and Marsh, T.J., 2008. High-flow and flood trends in a network of undisturbed catchments in the UK. Int. J. Climatol., 28, 1325-1338.

Hirsch, R.M. 2011. A perspective on nonstationarity and water management. J. Amer. Water Resour.Ass., 47, 436446, DOI: 10.1111/j.1752-1688.2011.00539.x.

Kjeldsen, T.R. 2010. Modelling the impact of urbanization on flood frequency relationships in the UK. Hydrol. Res., 41, 391-405, doi:10.2166/nh.2010.056.

Laio, F., Di Baldassarre, G. and Montanari, A. 2009.

Model selection techniques for the frequency analysis of hydrological extremes. Water Resour. Res., 45, W07416, 11 pp., doi:10.1029/2007WR006666.

Marsh, T. and Harvey, C.L. 2012. The Thames flood series: a lack of trend in flood magnitude and a decline in maximum levels. Hydrol. Res., 43, 203-214, doi:10.2166/nh.2012.054

Robson, A.J. 2002. Evidence for trends in UK flooding. Phil. Trans. R. Soc. London. A, 360, 1327-1343, doi: 10.1098/ rsta.2002.1003.

Vogel, R.M., Yaindl, C. and Walter, M. 2011. Nonstationarity: flood magnification and recurrence reduction factors in the United States. J. Amer. Water Resour. Ass., 47, 464-474, doi: 10.1111/j.1752-1688.2011.00541.x

Wheater, H. and Evans, E. 2009. Land use, water management and future flood risk. Land Use Policy, 26S, S251-S264, doi:10.1016/j.landusepol.2009.08.019.

Wilby, R.L., Beven, K.J. and Reynard, N.S. 2008. Climate change and fluvial flood risk in the UK: More of the same? Hydrol. Proc., 22, 2511-2523. 